\title{
A Giant Urethral Calculus
}

\author{
Ghanshyam Sigdel, ${ }^{1}$ Aman Agarwal, ${ }^{2}$ Belokar Wasant Keshaw ${ }^{3}$ \\ 'Department of Urology, College of Medical Sciences, Chitwan, Nepal, '2Department of Urology, College of Medical Sciences, \\ Chitwan, Nepal, ${ }^{3}$ Department of Urology, College of Medical Sciences, Chitwan, Nepal.
}

\section{ABSTRACT}

Urethral calculi are rare forms of urolithiasis. Majority of the calculi are migratory from urinary bladder or upper urinary tract. Primary urethral calculi usually occur in presence of urethral stricture or diverticulum. In this article we report a case of a giant posterior urethral calculus measuring $7 \times 3 \times 2 \mathrm{~cm}$ in a 47 years old male. Patient presented with acute retention of urine which was preceded by burning micturition and dribbling of urine for one week. The calculus was pushed in to the bladder through the cystoscope and was removed by suprapubic cystolithotomy.

Keywords: giant urethral calculus; posterior urethra.

\section{INTRODUCTION}

Urethral calculi represent only $0.3 \%$ of all urinary stone disease. ${ }^{1}$ Majority of them are small in size and present acutely with dysuria, dribbling or acute retention of urine. Giant urethral calculi are extremely rare. ${ }^{2}$ Larger size urethral calculi usually form in the background of preexisting urethral conditions like stricture or diverticulum. These calculi may remain silent for a long period of time or have less bothersome symptoms of dysuria or lower urinary tract symptoms. Here we present a case of a giant posterior urethral calculus and review of the literature.

\section{CASE REPORT}

A 42 years old gentleman presented with the complaints of acute retention of urine for several hours preceded by severe dysuria and dribbling of urine over a period of one week. There was no history of hematuria, fever, urethral trauma or instrumentation. On physical examination, bladder was distended. A hard, elongated, lump of $7 \times 4$ centimeter, with limited mobility was palpable in the perineum. X-ray pelvis revealed a large posterior urethral calculus. Laboratory parameters including urine culture, complete blood counts \& renal function test were normal. Ultrasonography of the abdomen and pelvis was normal. The stone was easily pushed in to the bladder with the cystoscope and subsequently removed through suprapubic cystolithotomy under spinal anesthesia. The posterior urethra was roomy but there was no evidence of urethral diverticulum or stricture .The stone measured $7.5 \times 4 \times 3$ centimeter and biochemical analysis demonstrated the presence of uric acid, amino acid, calcium and phosphate. Postoperative period was uneventful and patient voided normally.

\section{DISCUSSION}

Urethral calculi have a bimodal age distribution, with peak incidences in early childhood, as well as in the fourth decade of life..$^{3,4}$ Increased urinary peak flow rates may exert a protective effect in the second and third decades of life by allowing for increased clearance of calculi that migrate into the urethra, which may in part account for the relative paucity of urethral stone disease noted in this demographic group. . $^{3,4}$ The majority of urethral calculi occur in males and they are exceedingly rare in females because of low incidence of

Correspondence: Dr. Ghanshyam Sigdel, Department of Urology, College of Medical sciences Teaching Hospital, Chitwan, Nepal. E-mail: sigdelgs@yahoo.com, +977-9841219013. 
vesicle calculi and shorter urethra. ${ }^{6}$ Urethral calculi may result from migration from the bladder or upper tracts or may arise de novo. Large proportion of urethral calculi is migratory calculi that usually get impacted in the urethra due to preexisting lower urinary tract pathologic process, such as benign prostatic hyperplasia, urethral stricture, or meatal stenosis. In situ development of the urethral calculi primarily occurs through condensation of stone material on urethral foreign bodies or from stasis of urine in urethral diverticula. The clinical presentation of urethral calculus depends on pathogenesis and location of the stone within the urethra. The migratory calculi often present with acute onset of stranguary, dysuria, hematuria and urinary retention due to sudden impaction of the stone. Those patients with de novo urethral calculi and those residing in diverticula often present with more insidious symptoms. Kamal et al reported that 78 percent of all patients with urethral had acute retention of urine, while 22 percent reported decrease of the urinary stream with dribbling of urine. ${ }^{3}$ Urinary tract infection is frequently present at the time of presentation .

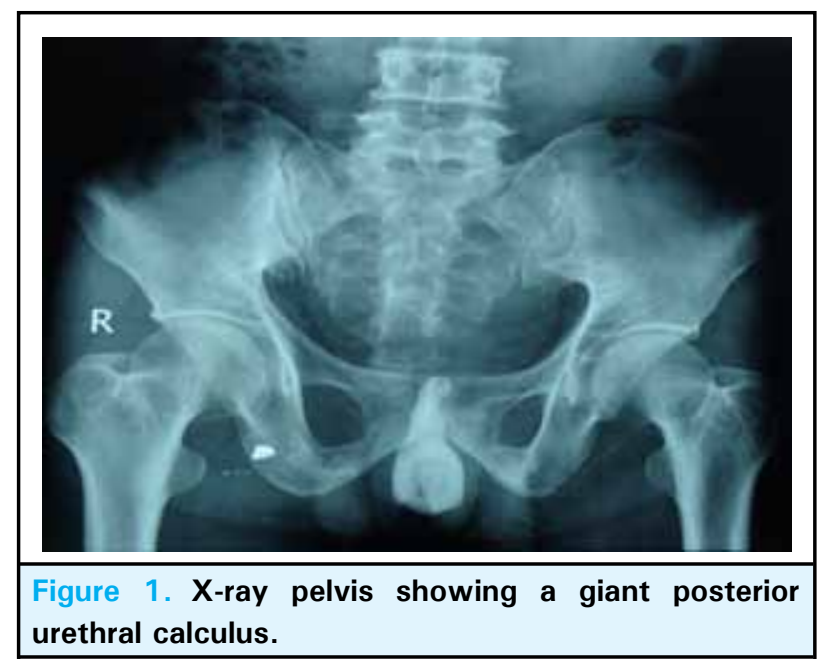

Thirty-two to 88 percent of the urethral calculi reside in the posterior urethra, whereas 8 percent to 58 percent are located in the bulbous and penile urethra and four percent to 11 percent are found at the fossa navicularis .$^{2,3}$ Ninety-eight to 100 percent of urethral calculi are radiopaque and can be visualized on plain radiographs
.3,4 Biochemically struvite stones predominate, although calcium phosphate and uric acid calculi have also been reported. $^{7}$

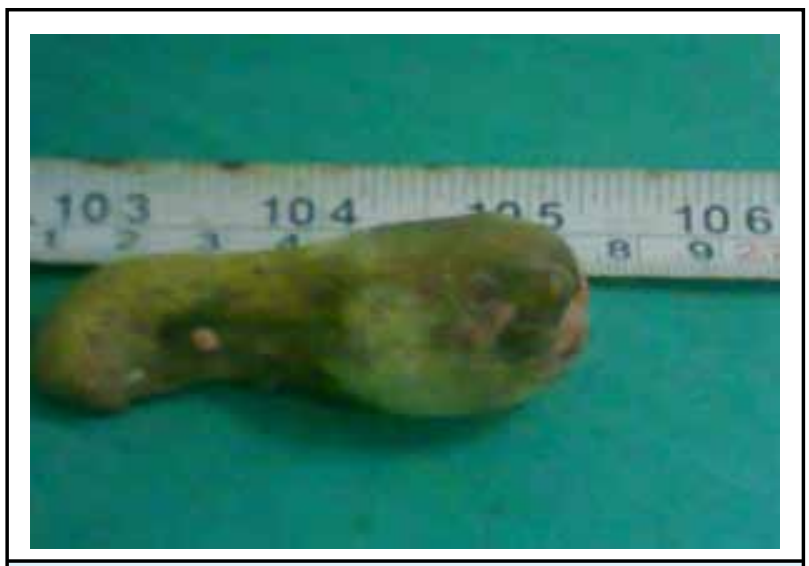

Figure 2. Removed giant urethral calculusmeasuring $7.5 \times 4 \times 3$ centimeter.

Treatment of urethral calculi depends on the size, location within the urethra and presence of an associated anatomic pathologic process, such as a diverticulua, stricture etc. Posterior urethral calculi may be pushed back into the bladder for subsequent intracorporeal or extracorporeal lithotripsy or open cystolithotomy with variable success rate..$^{3,4,8,9}$ In-situ lithotripsy of urethral calculi may be feasible, with reported success rates of up to $80 \% .{ }^{3}$ Lithotripsy can be done using electrohydrulic, pneumatic or laser energy source ; later being highly effective with minimal trauma to the surrounding urethral tissue. The treatment of choice for an impacted, large calculus in the bulbar urethra is perineal urethrotomy with urethroplasty.Anterior urehtral stone can be extracted by "milking", provided the stone is smooth. Urethrotomy with stone extraction is indicated for stones not amenable to simple manipulations or when concurrent urethroplasty or urethrocutaneous fistula repair is required. Stones within urethral diverticula are addressed with incision of the diverticulum, stone extraction followed by diverticulectomy and urethral repair in the index operation or in a staged fashion. In conclusion, giant urethral calculus is a rare entity and it can be managed with endoscopic or open surgical methods. 


\section{REFERENCES}

1. Aegukkatajit S. Reduction of urinary stone in children from north-eastern Thailand. J Med Assoc Thai. 1999; 82:1230-33.

2. Shanmugam TV, Dhanapal V, Rajaraman T, Chandrasekar CPM, Balashanmugam KP. Giant urethral calculi. Hospital Medicine.2000; 61:582.

3. Kamal BA, Anikwe RM, Darawani H, Hashish M, Taha SA. Urethral calculi: presentation and management.BJU Int. 2004; 93:549-52.

4. Verit A, Savas M, Ciftci H, Unal D, Yeni E, Kaya M. Outcomes of urethral calculi patients in an endemic region and an undiagnosed primary fossa navicularis calculus. Urol Res. 2006; 34: 37-40.

5. Jørgensen JB, Jensen KM. Uroflowmetry. Urol Clin North Am. 1996; 23:237-42.
6. Win T. Giant urethral calculus: Singapore Med J. 1994; 35:414-5.

7. Singh I, Neogi S. Male anterior urethral diverticula with Cobb's collar and a giant stone. J Post grad Med. 2006; 52:73-4.

8. Aus G, Bergdahl S, Hugosson J, Lundin SE. Stone formation in the prostatic urethra after cryotherapy for prostate cancer. Urology. 1997; 50: 615-7.

9. El-Sharif AE, Prasad K: Treatment of urethral stones by retrograde manipulation and extracorporeal shock wave lithotripsy. Br J Urol. 1995; 76:761-64. 